
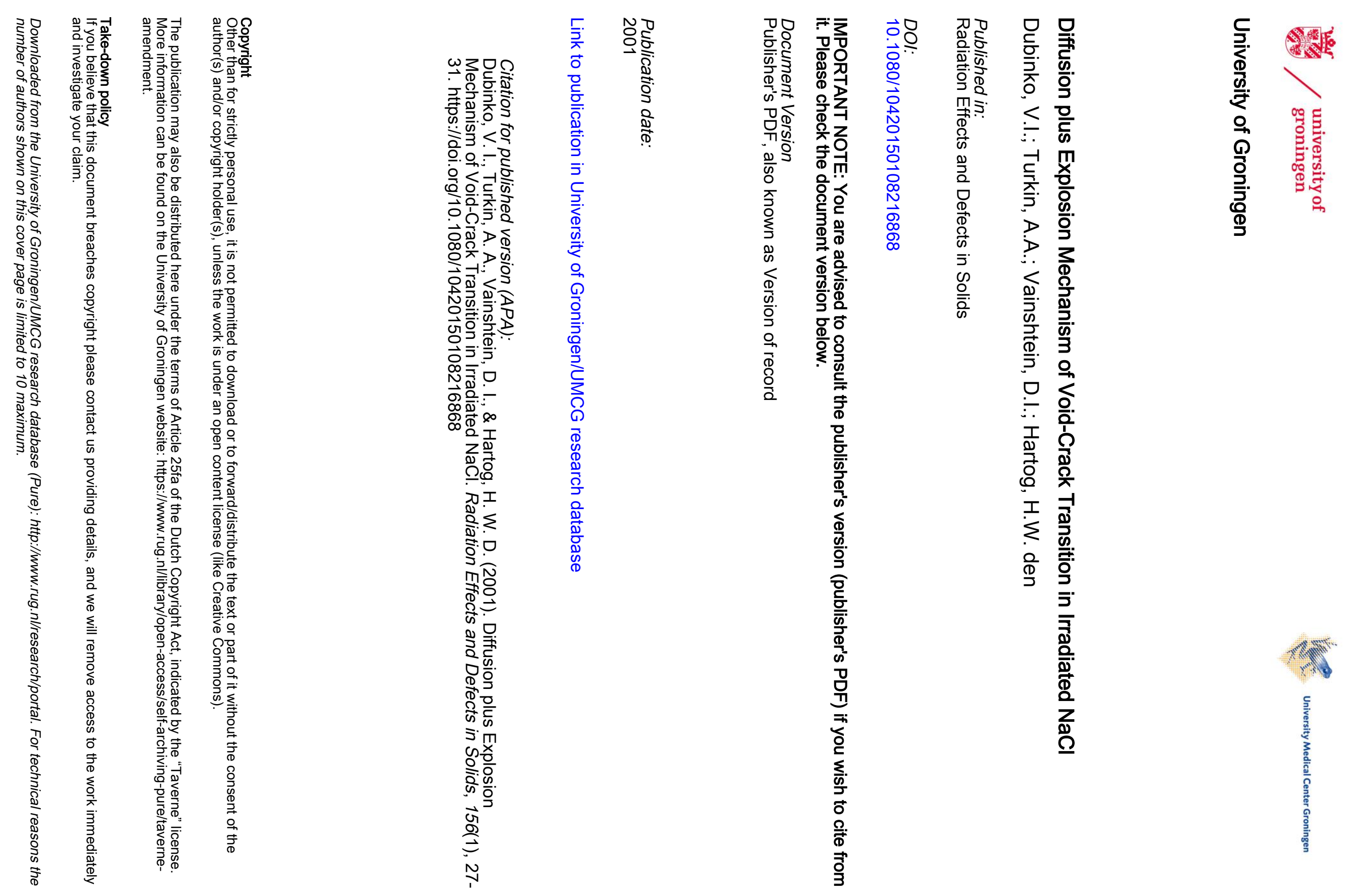


\title{
DIFFUSION PLUS EXPLOSION MECHANISM OF VOID-CRACK TRANSITION IN IRRADIATED NaCl
}

\author{
V.I. DUBINKO ${ }^{\mathrm{a}}$, A.A. TURKIN ${ }^{\mathrm{a}}$, D.I. VAINSHTEIN ${ }^{\mathrm{b}}$ \\ and H.W. den HARTOG ${ }^{\mathrm{b}, *}$ \\ ${ }^{a}$ Kharkov Institute of Physics \& Technology, 310108 Kharkov, Ukraine; \\ 'Solid State Physics Laboratory, University of Groningen 4 Nijenborgh, \\ NL-9747 AG Groningen, The Netherlands
}

(Received 7 April 2000; In final form 30 September 2000)

\begin{abstract}
Experimental and theoretical results are presented on the formation of large vacancy voids and cracks in $\mathrm{NaCl}$ under irradiation. The crack length increases gradually with increasing stored energy, which is explained by a new mechanism of diffusion accumulation and explosive release of energy in voids and cracks. It is shown that the colloid number density, which determines the critical void radius that triggers the explosion process, has a drastic effect on the ultimate mechanical stability of material. If it is low as in $\mathrm{NaCl}$ doped with $\mathrm{Br}$ then the critical void radius is large and difficult to reach. If it is high as in $\mathrm{NaCl}: \mathrm{KBF}_{4}$ it takes a high colloid volume fraction to initiate the void-crack transition, which progresses rather slowly. The materials doped with $\mathrm{Ba}$ or $\mathbf{K}$, and natural rock salts show the transitional behavior, which is characterized by an early start and a rapid progress of the void-crack transition, resulting in a subsequent fracture of the material.
\end{abstract}

Keywords: Radiation damage; Ionic crystals; Precipitates; Vacancy voids; Cracks.

\section{INTRODUCTION}

Ionizing irradiation of $\mathrm{NaCl}$ samples is known to result in the formation of chlorine "bubbles" (or rather solid or liquid chlorine precipitates, since the pressure in them is too high for the liquid-gas transition) formed by

\footnotetext{
* Corresponding author. E-mail: h.w.den.hartog@phys.rug.nl
} 
agglomeration of $H$ centers and of the complementary inclusions of sodium ("colloids") formed by agglomeration of $F$ centers $[1,2]$. The volume fraction of chlorine "bubbles" is closely related to that of sodium colloids, which is a measure of the stored energy. The higher it grows, the more potentially unstable becomes the material. But its actual mechanical stability is determined by a scenario of the stored energy release rather than by its value. If the back reaction quantum is small enough (as it is assumed in the Jain-Lidiard model [2], which considers only the recombination between chlorine molecules and $F$ centers) then it thay just limit the stored energy accumulation. But our experiments on heavily irradiated pure, doped and natural rock salt samples have shown that with increasing dose, formation of more or less round, sometimes faceted voids is observed followed by their transition to large penny-shaped cracks [3] (Fig. 1). We have proposed a new model $[4,5]$, in which voids are shown to arise due to the formation of electroneutral vacancy pairs at the surfaces of halo-
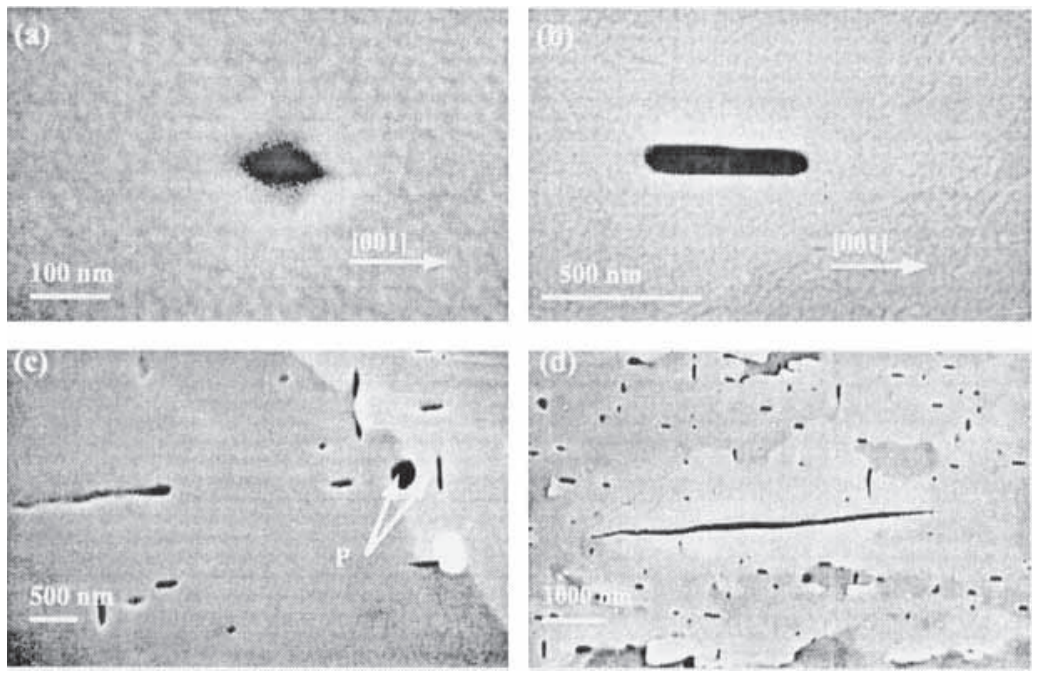

FIGURE 1 SEM micrographs showing vacancy voids and cracks in natural rock salt crystals irradiated with $0.5 \mathrm{MeV}$ electrons. (a) Equiaxial void at high resolution after irradiation to 60 Grad: latent heat of meiting (LHM) of metallic Na is $0.45 \mathrm{~J} / \mathrm{g}$. (b) "Penny-shaped" crack formed after irradiation to 300 Grad, LHM of metallic $N a$ is $0.8 \mathrm{~J} / \mathrm{g}$. (c) "Penny-shaped" voids/cracks in natural rock salt irradiated up to $300 \mathrm{Grad}$ : LHM of metallic $\mathrm{Na}$ is $1.2 \mathrm{~J} / \mathrm{g}$. The letter " $p$ " with the arrows shows two adjacent voids with different orientations. (d) Long crack formed after irradiation to $300 \mathrm{Grad}$, LHM of metallic Na is $2.5 \mathrm{~J} / \mathrm{g}$. 
gen bubbles and grow to sizes exceeding the mean distance, first, between bubbles and then between colloids, which changes the scale of the back reaction from the atomic to the macroscopic one. The voids absorb fine chlorine bubbles during their growth and accumulate a large number of chlorine molecules before the collision with colloids starts, which ignites a powerful back reaction resulting in an instantaneous pressure increase in a chlorine-filled void [3].

\section{DIFFUSION PLUS EXPLOSION MECHANISM OF VOID-CRACK TRANSITION}

The amount of released energy in the back reaction is proportional to the energy released due to the formation of one $\mathrm{NaCl}$ molecule, $\mathrm{q}_{\mathrm{NaCl}}$, and to the number of molecules formed as a result of the collision. The latter is equal to the mean number of sodium atoms in a colloid, which is close to the number of chlorine atoms accumulated in the void by the time of collision. The released energy heats up the reaction products inside the void resulting in an instantaneous temperature and pressure increase, which can be estimated as [3]

$$
\Delta T=\frac{2}{3} \frac{q_{\mathrm{NaCl}}}{k} \geq 2 \times 10^{4} K, \quad \Delta P \geq \frac{2}{3} \frac{q_{\mathrm{NaCl}}}{\omega} V_{C}
$$

where $k$ is the Boltzmann constant, $V_{C}$ is the colloid volume fraction and $\omega$ the atomic volume. So the pressure increase due to the back reaction is proportional to the colloid volume fraction, which mounts to pressures in the $\mathrm{GPa}$ range. Such a pressure may initiate crack propagation from the void along the matrix cleavage plane (100) if it is bigger than some threshold value [3] $P_{f}=\sigma_{f} / 2$, where $\sigma_{f}$ is the fracture stress to initiate the Griffith crack of the length equal the void diameter. Figure 1 shows the voidcrack evolution with increasing irradiation dose and the amount of stored energy in natural rock salt crystals. ${ }^{1}$ It is seen that the crack length increases

\footnotetext{
${ }^{1}$ The amount of stored energy is expressed in terms of latent heat of melting (LHM) of metallis $\mathrm{Na}$, which is proportional to the colloid volume fraction.
} 
gradually, which can be explained by a combined mechanism of diffusion accumulation and explosive release of energy in voids and cracks.

The final crack length is determined by the rates of two competing processes, namely, the production and dissipation of heat. Heat production is determined by the rate of crack expansion in length and thickness resulting in the consumption of a new "fuel" (chlorine and sodium precipitates), which increases the pressure by a factor proportional to the surface covered by the propagating crack, its thickness and the colloid volume fraction. The heat dissipation is determined mainly by the rate of propagation of the temperature front, within which the matrix atoms are evaporated at the expense of the energy released in the back reaction, which reduces the gas pressure. As a result, the crack propagation is stopped when the gas pressure falls below the threshold value. Accordingly, the final crack length is determined by the volume fraction and the number density of colloids, the crack propagation rate and material parameters, such as the themal conductivity and the heat of evaporation of $\mathrm{NaCl}$. The result is shown in Figure 2 in the form of crack length dependence on the colloid volume fraction at dif-

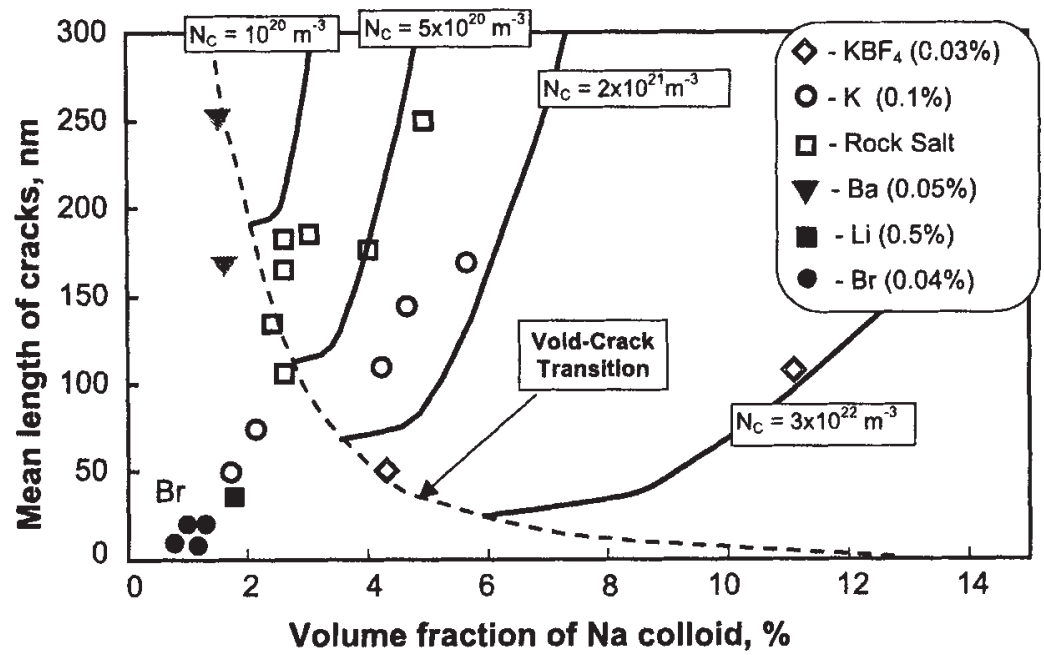

FIGURE 2 Diagram showing dependence of the maximum void dianeter and a subsequent crack length on the colloid volume fraction, calculated for different colloid number densities, $\mathrm{N}_{\mathrm{C}}$ indicated in the figure, assuming the crack thickness to be determined by the void diameter and its propagation rate to be equal to the speed of sound in $\mathrm{NaCl}$. Experimental points correspond to different dopants (concentrations are given in mol\%), for which experimental data on $N_{C}$ are presented in ref. $[6,7]$. 
ferent colloid number densities, expected for different dopants $[6,7]$ assuming the crack propagation rate to be equal to the speed of sound in $\mathrm{NaCl}$. It can be seen that the colloid number density, which determines the void explosion radius, has a drastic effect on the ultimate mechanical stability of material. If it is low as in $\mathrm{NaCl}: \mathrm{Br}$ (below $10^{21} \mathrm{~m}^{-3}$ ) then the void explosion radius is large and difficult to reach. If it is high as in $\mathrm{NaCl}: \mathrm{KBF}_{4}$ (above $10^{22} \mathrm{~m}^{-3}$ ), the void explosion radius is small, but it takes a high colloid volume fraction to initiate the void-crack transition, which progresses more gradually. The materials doped with $\mathrm{Ba}$ or $\mathrm{K}$, and natural rock salt show transitional behavior, which is characterized by an early start and a rapid progress of the void-crack transition, resulting in subsequent fracture of the material $[3,6,7]$.

\section{Acknowledgements}

This study is supported by the Dutch Ministry of Economic Affairs and by the NATO Linkage Grant CRG.LG 973314

\section{References}

[1] Lidiard, A.B. (1978) Comments Solid State Phys. 8, 73.

[2] Lidiard, A.B. (1979) Phil. Mag. 39, 647.

[3] Vainshtein, D.I., Dubinko, V.I., Turkin, A.A. and den Hartog, H.W. (2000) Nucl. Instr: and Meth. in Phys. Research, 166, 561.

[4] Dubinko, V.I., Turkin, A.A., Vainshtein, D.l. and den Hartog, H.W. (1999) J. Appl. Phys., 86, 5957.

[5] Dubinko, V.I., Turkin, A.A., Vainshtein, D.I. and den Hartog, H.W. (2000) J. Nucl. Mater. 227, 184.

[6] Vainshtein, D.I., den Hartog, H.W., Dubinko, V.I. and Turkin, A.A. Critical parameters controlling mechanical stability of $\mathrm{NaCl}$ tinder irradiation, submitted to ICDIM 2000.

[7] den Hartog, H.W., Vainshtein, D.I., Dubinko, V.I., Turkin, A.A., Gann V.V. and J. Jacobs, Radiation Damage in NaCl: Retrievability, Smart Backfill Materials, Monitoring, Final Report CORA Research Project for the Dutch Ministry of Economic Affairs, December 1999. 